\title{
DEPENDENCE OF THE SENSITIVITY TO PLANETS ON THE PROPERTIES OF HIGH-MAGNIFICATION GRAVITATIONAL MICROLENSING EVENTS
}

\author{
Cheongho Han \\ Department of Physics, Chungbuk National University, Cheongju 361-763, Korea \\ (Received June 13, 2011; Revised July 16, 2011; Accepted July 16. 2011)
}

\begin{abstract}
In current microlensing planet searches that are being carried out in a survey/follow-up mode, the most important targets for follow-up observations are lensing events with high magnifications resulting from the very close approach of background source stars to the lens. In this paper, we investigate the dependence of the sensitivity to planets on detailed properties of high-magnification events. From this, it is found that the sensitivity does not monotonically increase as the impact parameter between the lens and the source trajectory decreases. Instead, it is roughly the same for events with impact parameters less than a certain threshold value. It is also found that events involving main-sequence source stars are sensitive to planets in a much wider range of separation and mass ratio, than those events involved with giant source stars. Based on these results, we propose observational strategies for maximal planet detections considering the types of telescopes available for follow-up observations.
\end{abstract}

Key words : gravitational lensing: micro — binaries: general

\section{INTRODUCTION}

Microlensing is one of the most important methods that can detect and characterize extrasolar planets. The microlensing signal of a planet is a shortduration perturbation on the smooth and symmetric single-lensing light curve induced by the primary lens (Mao \& Paczynski 1991; Gould \& Loeb 1992). The duration of the perturbation is short, ranging from several days for giant planets to several hours for terrestrial planets. To detect short planetary signals, current microlensing experiments are being carried out in a survey/follow-up mode. In this mode, survey observations are designed to maximize the number of event detections by monitoring a large area of sky but with low-cadence coverage of events. Follow-up observations, on the other hand, are focused on events alerted by the survey observations to densely cover the events with multiple number of telescopes. However, the limited number of telescopes and time available for followup observations make it difficult to densely monitor all alerted events. As a result, follow-up observations are conducted for a fraction of events with high sensitivity to planets. Currently, the highest priority is given to high-magnification events. The source trajectories of these events pass close to the central perturbation region induced by a caustic, located near the lens hosting the planet and thus the sensitivity to planets is high (Griest \& Safizadeh 1998).

Although high, the sensitivity to planets detectable through the central-perturbation channel can vary depending on various factors. These factors include (1) density of coverage, (2) precision of data, (3) impact parameter between the lens and source trajectory, and (4) type of source stars. Among them, the first two factors are restricted by the instrument used for experiments, and thus, it is difficult to adjust the observational setup based on these factors. However, the other two factors are intrinsic to events and adjustment of observational strategy depending on these factors is possible to maximize planet detections.

In this paper, we investigate the sensitivity of highmagnification events to various types of planets depending on the properties of events such as the impact parameter and the type of source stars. Based on the results of this investigation, we propose observational strategies that can maximize planet detections with limited resources available for follow-up observations.

\section{CENTRAL PERTURBATONS}

The ultimate reason for the planetary microlensing perturbation is the formation of caustics in lens systems composed of multiple lens components. Caustics represent the positions on the source plane at which the lensing magnification of a point source is infinity. The set of caustics form several sets of closed curves each of which is composed of concave curves (folds) that meet at points (cusps). For planetary lensing, two sets of caustics are formed, one of which is located close to the primary lens (central caustic) and the other, away from the primary (planetary caustic). As a result, while perturbations induced by the planetary caustic can occur at any part of the light curve, perturbations induced by the central caustic always occur near the peak of high-magnification events. 

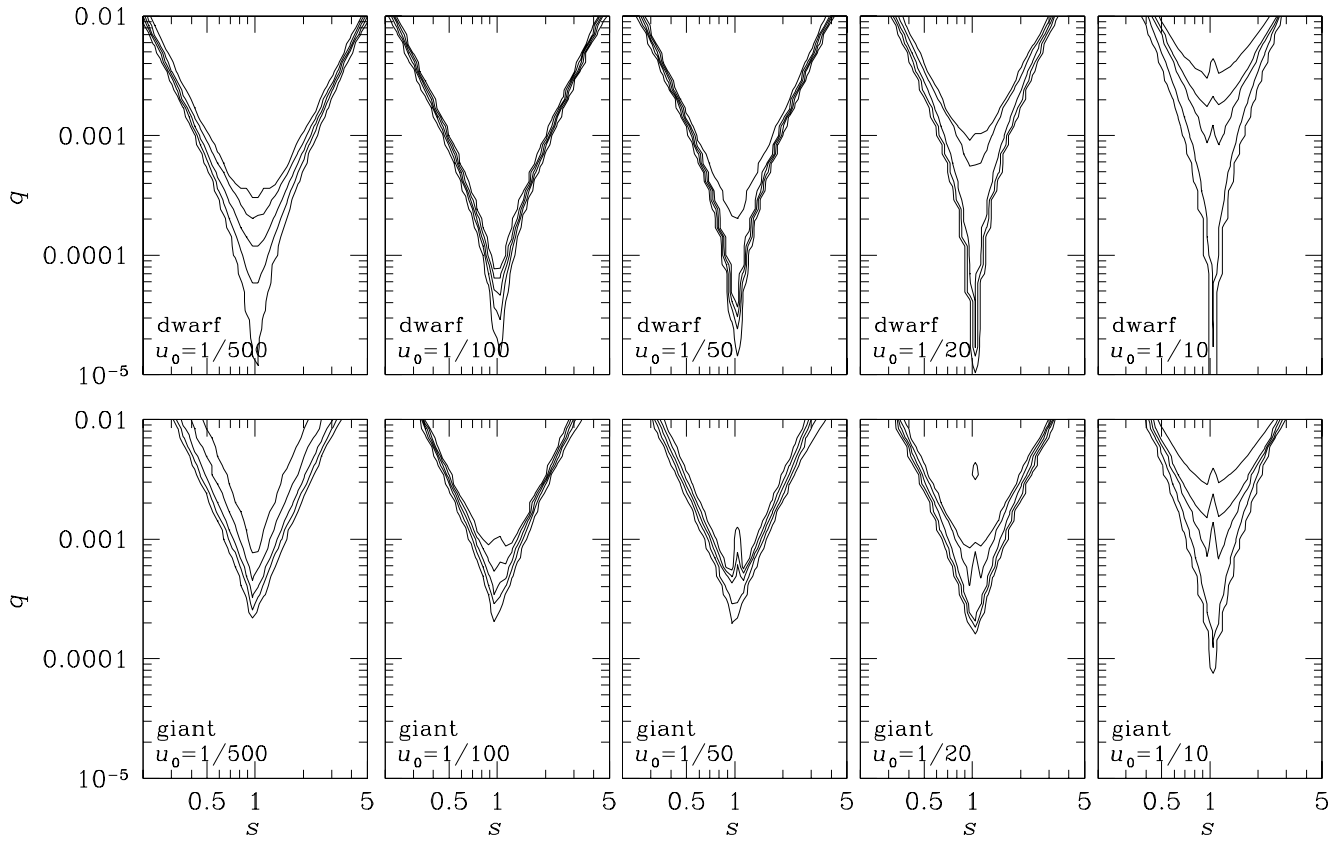

Fig. 1.- Microlensing sensitivity of high-magnification events to planets of various types presented as contour plots of the detection efficiency with respect to the projected star-planet separation $s$ and the planet/star mass ratio $q$ for various impact parameters $u_{0}$ and source type. The upper panels are for events associated with a dwarf source star, while the lower panels are for events with a giant star. The contours are drawn at the levels of $10 \%, 30 \%, 50 \%, 70 \%$, and $90 \%$, respectively.

The central caustic has a wedge shape with 4 cusps, two of which are located on the star-planet axis and two more off the axis. It axially elongated and its size measured as the separation between the two on-axis cusps is (Chung et al. 2005)

$$
\Delta \xi=\frac{4 q}{\left(s-s^{-1}\right)^{2}},
$$

where $q$ is the planet/star mass ratio and $s$ is the projected star-planet separation in units of the Einstein radius, $\theta_{\mathrm{E}}$, corresponding to the total mass of the lens system. The caustic size becomes maximum when the planet is located near the Einstein ring of the primary, i.e., $s \sim 1$ and decreases as $s^{-2}$ for planets with $s \gg 1$ and as $s^{2}$ for planets with $s \ll 1$. The caustic size also depends on the planet/star mass ratio such that it increases in linear proportion to the mass ratio. For a given mass ratio, a pair of central caustics with separations $s$ and $s^{-1}$ are identical to the first order of approximation (Dominik 1999; An 2005). The caustic size corresponds to the cross-section of the planetary perturbation. Therefore, the microlensing sensitivity varies depending on the planetary separation and mass ratio.

Besides the factors intrinsic to the lens system, the sensitivity to planets depends also on the source type. The source type affects the sensitivity in two different ways. The first effect is caused by the finite size of the source star. The magnification of a finite source corresponds to the intensity-weighted mean averaged over the source star flux. Therefore, the finite size of the source weakens planetary signals by smoothing and broadening the caustic-induced perturbations (Bennett \& Rhie 1996; Gould \& Gaucherel 1997). Finite-source effect depends on the angular size of the source star, $\theta_{\star}$, in units of the Einstein radius, i.e., $\rho_{\star}=\theta_{\star} / \theta_{\mathrm{E}}$. The second effect of the source type on the sensitivity is caused by the photometric precision which depends on the source brightness. However, this effect is less critical for high-magnification events because source stars during central perturbations are bright enough for precision photometry regardless of the source type.

\section{SENSITIVITY}

We investigate dependence of the sensitivity of high-magnification events to planets on the impact parameter* and the source type according to the follow-

\footnotetext{
*The dependency on the impact parameter instead of the peak magnification is investigated for 2 reasons. First, the impact parameter is more directly related to the planet detection efficiency because it describes how closely the source approaches the central perturbation region. On the other hand, the peak magnification additionally depends on the source type and thus
} 

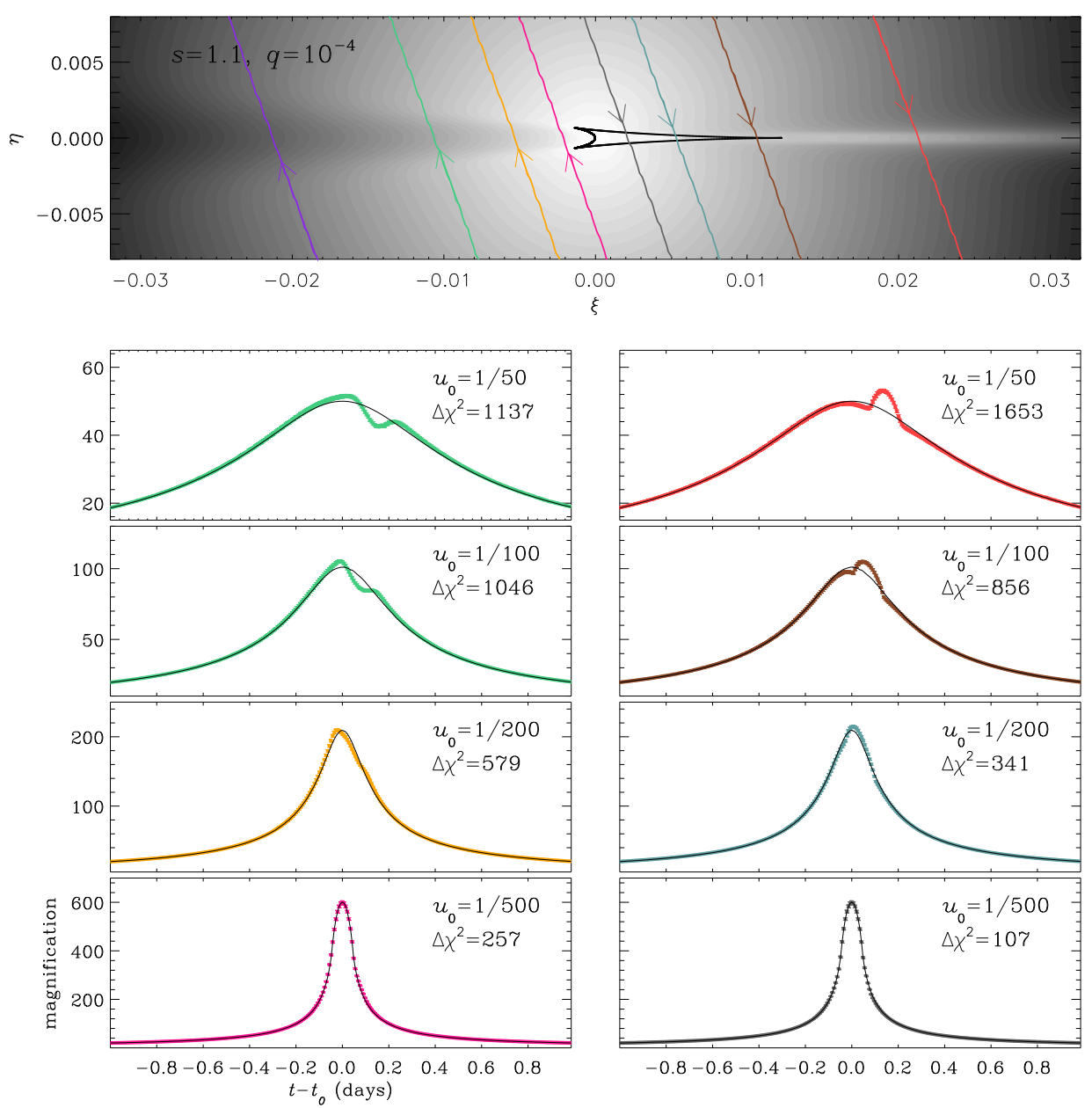

Fig. 2.- Light curves of planetary events resulting from various source trajectories with respect to the central caustic of an example planetary lens system with $s=1.1$ and $q=10^{-4}$. The color of each light curve corresponds to that of the source trajectory in the top panel where the lens system geometry is presented. The black curve in each panel represents the best-fit single-lens light curve. The values in each panel are the impact parameter, $A_{\max }$, and $\Delta \chi^{2}$ between the planetary and single-lensing light curves. The adopted source radius normalized by the Einstein radius is $\rho_{\star}=0.003$. Presented are the light curves for 2 days around the time of the closest source-primary approach, $t_{0}$.

ing procedure. For events with a given impact parameter produced by a planetary system characterized by $s$ and $q$, we produce a large number of planetary lensing light curves resulting from source trajectories with different source trajectory angles with respect to the star-planet axis, $\alpha$. We estimate the planet detection efficiency as the fraction of events with detectable planetary signals among the total number of tested events.

does not directly describe the geometric alignment between the lens and source. Second, alerts of high-magnification events are based on the impact parameter determined from modeling of the rising part of the light curve even before the peak.
For this estimation, we use a criterion of $\Delta \chi^{2} \geq 500$ to judge the detectability of planets. The best-fit singlelens light curves corresponding to the light curves of the individual planetary events are obtained by considering the offset of the magnification center between the planetary and single-lens light curves. Central perturbations occur while the source star is highly magnified. Hence we assume that the photometric precision is dominated by the systematic error rather than photometric error. The adopted precision during the perturbation is $1 \%$. We also assume that the peak of the light curve is uniformly covered with a cadence of 
every 10 minutes during 2 days at the peak.

In producing light curves, we take the finite-source effect into consideration. Computing precise finite source magnifications is computationally very expensive. Considering that for sensitivity estimation one requires to produce a large number of light curves, it is important to use a robust and efficient method. We compute finite-source magnifications by using the rayshooting method. In this method, a large number of rays are uniformly shot into the image plane, bent according to the lens equation, and collected in the source plane. The magnification is computed as the ratio of the number density of rays that have arrived on the source star surface to those of the image plane. On the basis of this scheme, we minimized the amount of computation by using several methods. Firstly, we made a single map of rays in the source plane for a given set of $s$ and $q$ and used it to produce many light curves resulting from different values of the lens-source impact parameter and source trajectory angle. In this procedure, the locations of the individual rays which arrived on the source plane are stored in the memory of a computer so that they can be readily used for magnification computations. Secondly, the number of rays needed for the construction of a map wass minimized by restricting the region of the map only to the central part as the central perturbations occur only in this region. The central region in the source plane corresponds to the interior of an annulus encompassing the Einstein ring of the primary lens. Therefore, rays are shot only in this annulus region. Thirdly, even when the source position is within the central region, we restricted magnification computations based on the numerical ray-shooting method to the case when only the source is close to the caustic. When the source position is far enough from the caustic, we use a semianalytic approximation (Gould 2008). Our method of finite-source computations is similar to that developed by Dong et al. (2006) and Bennett (2010), although the details are different. In our computation, we test the normalized source radii of $\rho_{\star}=0.02$ and 0.003 , which are the typical values of Galactic bulge events involved with giant and dwarf source stars, respectively.

Fig. 1 shows the obtained sensitivity to planets presented as contour plots of the planet detection efficiency with respect to $s$ and $q$ for various impact parameters and different source types. The impact parameter $u_{0}$ is expressed in units of the Einstein radius. The contours form an overturned triangular shape where the apex is located at the separation $s \sim 1$ and a certain minimum mass ratio and its sides broaden with increasing $q$. Therefore, the diagram is often called the "triangle diagram". From the figure, we find the following trends in the sensitivity.

1. It is found that the sensitivity does not monotonically increase with the decrease of the impact parameter. Instead, it is roughly the same for events with impact parameters less than a thresh- old value of $u_{0} \sim 1 / 50$.

2 . In contrast to the dependence on $u_{0}$, the sensitivity depends greatly on the source type. It is found that events with main-sequence source stars are sensitive to planets with substantially wider range of separation and mass ratio.

Considering the dependence of the caustics size on $s$ and $q$, naive thinking leads to the expectation that events with smaller impact parameter would have a higher chance to be perturbed because the source approaches closer to the caustic. We find that the discrepancy between the expectation and the result is explained by the characteristic perturbation pattern around planet-induced central caustics. The pattern is such that perturbations are not confined to a region within the vicinity of the caustic but stretches out into considerably extended regions along the starplanet axis. Although the strength of the perturbation diminishes with increase in the distance to the caustic, the region of perturbation broadens. Therefore, the decrease of the contribution to $\Delta \chi^{2}$ by the weakened perturbation strength is compensated by the increased duration of the perturbation. As a result, the sensitivity dependency on the impact parameter is weak. To demonstrate this, in Fig. 2, we present several light curves with central perturbations resulting from source trajectories with different impact parameters. It shows that the perturbation of the event with $u_{0}=1 / 50$ is not much different from that of the event with $u_{0}=1 / 100$ and even stronger than the perturbation of the event with $u_{0}=1 / 500$. For events with $u_{0}=1 / 50$, the source trajectory passes through the extended perturbation region beyond the caustic and thus the resulting perturbation lasts for a considerable duration. On the other hand, the trajectory of the event with $u_{0}=1 / 500$ passes through the slim perturbation region formed in the very vicinity of the elongated caustic and thus the perturbation is short. In addition, non-negligible source size compared to the caustic size, even for a main-sequence star, makes the perturbation weak.

We check the consistency of our results by comparing them with the results obtained from the analyses of actually observed high-magnification events. In Table 1 , we present the list of events for which the sensitivity analyses similar to ours were conducted by Gould et al. (2010). The events are listed according to the order of sensitivity, implying that events with sensitivities to planets in wider ranges of $s$ and $q$ appear earlier in the list. We note that among the 11 events analyzed by Gould et al. (2010), 5 events with measured source radii are listed to see the dependence of the sensitivity on the source type. From the table, it is found that the order of sensitivity has little correlation with the impact parameter, supporting our result of the weak dependence of the sensitivity on $u_{0}$. On the other hand, the sensitivity order is exactly identical to the order of the source radius. This also supports our 
result of the strong dependence of the sensitivity on the source type.

\section{DISCUSSION}

The results of the dependencies of the sensitivity on the impact parameter and source type can be used as important criteria in selecting target events for followup observations and thus for the establishment of observational strategies for maximal planet detections under various circumstances.

In current lensing experiments, the telescopes participating in follow-up observations are broadly divided into 3 categories, depending on the availability of telescopes. Telescopes in the first category are exclusively used for lensing experiments. Telescopes in the second category are used partially in the way of targetof-opportunity. Telescopes in the third category are operated by amateur astronomers.

For telescopes in the second category, it is difficult to conduct extended follow-up observations for many events. The situation is similar for telescopes in the third category because the operators of the telescopes work during day time and thus it is difficult for them to observe too many target events or for too long a duration, inspite of their immense devotion to the experiment. For these telescopes, events with very low impact parameters are good targets, for two reasons. Firstly, the frequency of these events is not very high. Secondly, the required duration of follow-up observations, characterized by the full-width-half-maximum of the light curve, is linearly proportional to the impact parameter, i.e., FWHM $\sim 2 u_{0} t_{\mathrm{E}}$, and thus short.

Telescopes in the first category can be used to monitor events with moderately low impact parameters. These telescopes are often idle due to the absence of proper target events. With extensive operation of the telescopes by reducing the threshold impact parameter for follow-up observations, it will be possible to increase the number of planet detections. The low sensitivity to planets for events involved with giant source stars implies that it is important to consider not only peak magnification but also the source type in the selection of target events for follow-up observations.

The results of our work are also important for the setup of the observational strategies for future lensing experiments, that will survey fields with cadences

Table 1.

High-magnification events

\begin{tabular}{ccc}
\hline \hline event & $\log \left(u_{0}\right)$ & $\log \left(\rho_{\star}\right)$ \\
\hline OGLE-2006-BLG-109 & -2.5 & -3.5 \\
OGLE-2005-BLG-169 & -2.9 & -3.4 \\
OGLE-2007-BLG-349 & -2.7 & -3.3 \\
MOA-2007-BLG-400 & -3.6 & -2.5 \\
MOA-2008-BLG-310 & -2.5 & -2.3 \\
\hline
\end{tabular}

that are nearly two orders higher than those of the current surveys. Korea Microlensing Telescope Network (KMTNet) is such a survey that will achieve a 10 minute sampling rate by using a network of three 1.6 $\mathrm{m}$ telescopes to be exclusively used for microlensing. With increased cadence, planets can be detected by the survey itself without follow-up observations. Among these planets, a significant fraction will be detected through the channel of central perturbations. Therefore, it is important to set up observational strategies that can maximize planet detections through this channel. For example, the exposure time could be set so that moderately high-magnification events associated with main-sequence source stars are not saturated at their peaks.

\section{ACKNOWLEDGMENTS}

This work is supported by the 2010 grant of Chungbuk National University.

\section{REFERENCES}

An, J. H. 2005, Gravitational Lens under Perturbations: Symmetry of Perturbing Potentials with Invariant Caustics, MNRAS, 356, 1409

Bennett, D. P. 2010, An Efficient Method for Modeling High-magnification Planetary Microlensing Events, ApJ, 716, 1408

Bennett, D. P., \& Rhie, S. H. 1996, Detecting EarthMass Planets with Gravitational Microlensing, ApJ, 472,660

Chung, S.-J., et al. 2005, Properties of Central Caustics in Planetary Microlensing, ApJ, 630, 535

Dominik, M. 1999, Ambiguities in FITS of Observed Binary Lens Galactic Microlensing Events, A\&A, 341, 943

Dong, S., et al. 2006, Planetary Detection Efficiency of the Magnification 3000 Microlensing Event OGLE2004-BLG-343, ApJ, 642, 842

Gould, A. 2008, Hexadecapole Approximation in Planetary Microlensing, ApJ, 681, 1593

Gould, A., \& Gaucherel, C. 1997, Stokes's Theorem Applied to Microlensing of Finite Sources, ApJ, 477, 580

Gould, A., \& Loeb, A. 1992, Discovering Planetary Systems through Gravitational Microlenses, ApJ, 396, 104

Gould, A., et al. 2010, Frequency of Solar-like Systems and of Ice and Gas Giants Beyond the Snow Line from High-magnification Microlensing Events in 2005-2008, ApJ, 720, 1073

Griest, K., \& Safizadeh, N. 1998, The Use of HighMagnification Microlensing Events in Discovering Extrasolar Planets, ApJ, 500, 37

Mao, S., \& Paczynski, B. 1991, Gravitational Microlensing by Double Stars and Planetary Systems, ApJ, 374, 37 